\title{
Standing Waves in One-Dimensional Resonator Containing an Ideal Isothermal Gas Affected by the Constant Mass Force
}

\author{
Anna PERELOMOVA \\ Gdansk University of Technology \\ Faculty of Applied Physics and Mathematics \\ Narutowicza 11/12, 80-233 Gdańsk, Poland; e-mail: anpe@mif.pg.gda.pl \\ (received July 24, 2016; accepted February 7, 2017)
}

\begin{abstract}
The study is devoted to standing acoustic waves in one-dimensional planar resonator which containing an ideal gas. A gas is affected by the constant mass force. Two types of physically justified boundary conditions are considered: zero velocity or zero excess pressure at both boundaries. The variety of nodal and antinodal points is determined. The conclusion is that the nodes of pressure and antinodes of velocity do not longer coincide, as well as antinodes of pressure and nodes of velocity. The entropy mode may contribute to the total field in a resonator. It is no longer isobaric, in contrast to the case when the external force is absent. Examples of perturbations inherent to the entropy mode in the volume of a resonator are discussed.
\end{abstract}

Keywords: acoustic resonator; non-uniform media; standing waves.

PACS No. 43.28-g, 47.55.Hg.

\section{Introduction}

External forces which are applied to a fluid cause its density, temperature, and pressure to be some functions on spatial coordinates, and probably, on time. They may accelerate fluid ensuring the bulk flows of it. This essentially complicates the definition of specific perturbations (acoustic and other ones) of infinitely small magnitude which may exist in a fluid. Types of the wave motion are usually determined by dispersion relations. The dispersion relations, in turn, follow from the system of conservation equations in a fluid with account for external forces and sources of energy. Even in the simplest case of a planar one-dimensional flow affected by an external stationary force, the dispersion relations may be exactly introduced over all wave-length range of perturbations in some special cases only. This is conditioned by choice of new variables which provide constant coefficients by their partial derivatives in all conservation equations. The gravity force with constant acceleration is one of these cases. It makes the background pressure and density of an isothermal ideal fluid depend exponentially on the vertical coordinate (Eckart, 1960; Pedloski, 2006). Another example is a non-inertial or inertial constant mass force. In one dimension, there exist three types of motion: two acoustic modes and the entropy, non-wave mode correspondent to zero frequency. The entropy mode is stationary if the thermal conduction of a fluid is ignored, otherwise, its evolution in time is described by the diffusity equation (Brekhovskikh, Godin, 1990; Chu, Kovasznay, 1958; Rudenko, Soluyan, 1977). In the flows going out one dimension, the buoyancy waves appear (ECKART, 1960; BREKHOVSKIKH, Godin, 1990; Jones, 2001). They are of importance in the Earth meteorology (ECKART, 1960; PEDLOSKI, 2006; Brekhovskikh, Godin, 1990) and the planetary atmosphere dynamics applications.

Some recent analytical methods in evaluations of energetic contributions of different modes are fairly successful. This concerns also flows where dispersion relations cannot be introduced (LEBLE, 1990). These methods usually start from establishment of the relationships between perturbations inherent to every mode (so-called polarisation relations) (LEBLE, Perelomova, 2013; Perelomova, 2006). The proper conditions which correspond to the basic physical foundations should be applied at the boundaries of a volume. The boundary conditions cannot be arbitrary, 
they must follow from the conservation of the total energy in the whole volume of a resonator. In addition to travelling waves, the entropy mode may participate in the total field in a volume of a resonator. In view of that, the knowledge of travelling waves and non-wave motions, establishment of polarisation relations and boundary conditions should be necessary issues in description of standing waves also in fluids affected by external forces. Linear standing waves (that is, waves with perturbations of infinitely small magnitude) in the most simple case of one-dimensional resonator filled with a uniform ideal fluid in the absence of the external forces are well-studied. The entropy mode in this case represents constant isobaric increase or decrease in temperature in the whole volume, which in turn affects the sound speed and, by means of that, spacial spectrum of standing waves.

\section{Conservation equations and dispersion relations}

The equations governing inviscid and nonconducting fluid manifest conservation of momentum, energy, and mass. They determine dynamics of all possible types of motion which may take place in a fluid. We start from the linearised conservation equations in terms of excess pressure and density, $p^{\prime}$ and $\rho^{\prime}$ (the stationary background quantities $\bar{p}, \bar{\rho}$ are functions of coordinates):

$$
\begin{aligned}
& \frac{\partial \mathbf{V}}{\partial t}=-\frac{\nabla p^{\prime}}{\bar{\rho}}+\mathbf{g} \frac{\rho^{\prime}}{\bar{\rho}}, \\
& \frac{\partial p^{\prime}}{\partial t}=-\mathbf{V} \cdot(\nabla \bar{p})-\gamma \bar{p}(\boldsymbol{\nabla} \cdot \mathbf{V}), \\
& \frac{\partial \rho^{\prime}}{\partial t}=-\mathbf{V} \cdot(\nabla \bar{\rho})-\bar{\rho}(\boldsymbol{\nabla} \cdot \mathbf{V}),
\end{aligned}
$$

where $\mathbf{V}$ is the fluid's velocity, $\gamma=C_{p} / C_{v}$ designates the specific heats ratio, and $C_{p}, C_{v}$ are heat capacities under constant pressure and volume, respectively. The mass force is designated by the constant vector $\mathbf{g}=$ $(0,0,-g)$. It may represent gravitational acceleration or other constant mass force. It may also represent a non-inertial force in the reference frame in which fluid is motionless in equilibrium. A flow of an ideal gas is considered, whose internal energy $e$ in terms of the total pressure and density takes the form

$$
e=\frac{p}{(\gamma-1) \rho}
$$

The background pressure and density are determined by the zero order equality,

$$
\frac{\mathrm{d} \bar{p}(z)}{\mathrm{d} z}=-g \bar{\rho}(z)
$$

The functions providing constant temperature $T_{0}$ of the background ensure constant internal energy as well. They are well-established:

$$
\begin{aligned}
& \bar{p}(z)=p_{0} \exp (-z / H)=\rho_{0} g H \exp (-z / H) \\
& \rho(z)=\rho_{0} \exp (-z / H)
\end{aligned}
$$

where $H$ is the scale of homogeneity of a gas (ECKART, 1960; PEDLOSKI, 2006; BREKHOVSKIKH, Godin, 1990):

$$
H=\frac{T_{0}\left(C_{p}-C_{v}\right)}{g}
$$

and $p_{0}=\bar{p}(0), \rho_{0}=\bar{\rho}(0)$.

In the further analysis, we will consider onedimensional fluid's motion along the vertical axis with one component of velocity, $V$. All perturbations are functions of $z$ and $t$. It is convenient to make use of the quantity $\phi^{\prime}$ instead of perturbation in density,

$$
\phi^{\prime}=p^{\prime}-\gamma \frac{\bar{p}}{\bar{\rho}} \rho^{\prime}
$$

The reasons for this choice are listed at the end of the current section. Equations (1), rearranged in the new set of variables (LEBle, Perelomova, 2013),

$$
\begin{aligned}
& P=p^{\prime} \cdot \exp (z / 2 H), \\
& \Phi=\phi^{\prime} \cdot \exp (z / 2 H), \\
& U=V \cdot \exp (-z / 2 H),
\end{aligned}
$$

take the form

$$
\begin{aligned}
& \frac{\partial U}{\partial t}=\frac{1}{\rho_{0}}\left(\frac{\gamma-2}{2 \gamma H}-\frac{\partial}{\partial z}\right) P+\frac{\Phi}{\gamma H \rho_{0}} \\
& \frac{\partial P}{\partial t}=-\gamma g H \rho_{0} \frac{\partial U}{\partial z}-g \rho_{0} \frac{\gamma-2}{2} U \\
& \frac{\partial \Phi}{\partial t}=-(\gamma-1) \rho_{0} g U
\end{aligned}
$$

The set of new variables established by Eqs. (7) possesses the important property. In contrast to initial Eqs. (1), the system Eqs. (8) consists of equations in partial derivatives with constant coefficients. That means that it establishes dispersion relations in the whole domain of the wavenumbers. In turn, dispersion relations determine types of fluid motion which may exist in the total flow. As usual, all variables $(U, P$ and $\Phi)$ are imposed to be the Fourier integrals of planar waves with specific amplitude, frequency $\omega$, and wavenumber $k$ each: 


$$
\begin{aligned}
& U=\int_{-\infty}^{\infty} \widetilde{U} \exp (i \omega t-i k z) \mathrm{d} k, \\
& P=\int_{-\infty}^{\infty} \widetilde{P} \exp (i \omega t-i k z) \mathrm{d} k, \\
& \Phi=\int_{-\infty}^{\infty} \widetilde{\Phi} \exp (i \omega t-i k z) \mathrm{d} k .
\end{aligned}
$$

This yields the dispersion equation, that is, an algebraic equation which reflects the solvability of Eqs. (8) in terms of amplitudes of individual planar waves $\widetilde{U}$, $\widetilde{P}$, and $\widetilde{\Phi}$. That imposes the main determinant of the system being equal to zero. The roots of dispersion equation represent the dispersion relations:

$$
\begin{gathered}
\omega_{0}=0, \quad \omega_{1}=\sqrt{\gamma g H} \sqrt{k^{2}+\frac{1}{4 H^{2}}}, \\
\omega_{2}=-\sqrt{\gamma g H} \sqrt{k^{2}+\frac{1}{4 H^{2}}} .
\end{gathered}
$$

Two last roots are acoustic and relate to perturbations propagating in the positive and negative directions of axis $O Z$, and the first one (indexed by zero) relates to the stationary perturbations. It determines the nonwave mode, called entropy or thermal mode of a fluid's motion. The dispersion relations determine links which connect amplitudes of the planar waves for any type of motion. For example, relations for $\widetilde{P}$ and $\widetilde{\Phi}$ for both acoustic modes take the form:

$$
\widetilde{P}_{1,2}=\frac{1}{\gamma-1}\left(\frac{\gamma-2}{2}-i k \gamma H\right) \widetilde{\Phi}_{1,2} .
$$

This establishes the links of $P(z, t)$ and $\Phi(z, t)$ in both acoustic branches in accordance to Eqs. (9) at any instant and any coordinate,

$$
P_{1,2}=\frac{1}{\gamma-1}\left(\frac{\gamma-2}{2}+\gamma H \frac{\partial}{\partial z}\right) \Phi_{1,2} .
$$

The link for the perturbations in the stationary entropy mode takes the form

$$
\Phi_{0}=\left(-\frac{\gamma-2}{2}+\gamma H \frac{\partial}{\partial z}\right) P_{0} .
$$

The choice of variable $\phi$ instead of perturbation in density $\rho^{\prime}$ is reasoned by the following arguments. Firstly, $\phi^{\prime}$ measures the deviation of the thermodynamic process from the isentropic one. The variation in specific entropy $s$ of the fluid's element in unit time along its trajectory is determined by the equality

$$
T \frac{\mathrm{d} s}{\mathrm{~d} t}=\frac{\mathrm{d} e}{\mathrm{~d} t}-\frac{p}{\rho^{2}} \frac{\mathrm{d} \rho}{\mathrm{d} t}
$$

which in the case of infinitely-small magnitude flow of an ideal gas in the absence of an external force $(g=0)$ rearranges as

$$
T_{0} \frac{\partial s^{\prime}}{\partial t}=\frac{\frac{\partial p^{\prime}}{\partial t}-\frac{\gamma p_{0}}{\rho_{0}} \frac{\partial \rho^{\prime}}{\partial t}}{\rho_{0}(\gamma-1)}=\frac{1}{\rho_{0}(\gamma-1)} \frac{\partial \phi^{\prime}}{\partial t} .
$$

This provides equality $\phi^{\prime}=0$ for the isentropic processes with zero local variation in entropy, $s^{\prime}=0$. The acoustic part of $\phi^{\prime}$ is proportional to the perturbation in acoustic entropy, $\phi_{a}^{\prime}=(\gamma-1) \rho_{0} T_{0} s_{a}^{\prime}$. It is identically zero in both isentropic acoustic branches. In the case of non-zero mass force, in contrast to the case $g=0$, the non-zero $\Phi_{a}\left(\Phi_{a}=\Phi_{1}+\Phi_{2}\right)$ may represent perturbation of $\phi_{a}$. It is connected with $P_{a}\left(P_{a}=P_{1}+P_{2}\right)$ by means of Eq. (12). The simple link between acoustic perturbations Eq. (12) is local. That is the second reason to choose variable $\phi$ instead of density $\rho$. The relation between $P$ and $R$ (where $R=\rho^{\prime} \exp (z / 2 H)$ ) has been derived by the author in (PERElomova, 1998). It is integro-differential with some kernel which reflects dispersive properties of sound in a stratified medium. In contrast to acoustic waves, the entropy mode posseses stationary perturbation in entropy. In the flows without external force, the entropy mode is isobaric with any smooth perturbation of mass density, but if $g \neq 0$, it is not, in accordance to the relation between $\Phi_{0}$ and $P_{0}$, Eq. (13).

\section{Perturbations for different kinds of boundary conditions}

The total energy of a gas $\varepsilon$ in a volume of a cylinder of a cross-sectional area $S$ which is bounded by planes $z=0$ and $z=L$, consists of kinetic, barotropic, and thermal contributions (Leble, Perelomova, 2013):

$$
\begin{aligned}
\varepsilon & =\frac{S}{2} \int_{0}^{L} \mathrm{~d} z\left(\bar{\rho} V^{2}+\frac{p^{\prime 2}}{\gamma \bar{p}}+\frac{\phi^{2}}{\gamma(\gamma-1) \bar{p}}\right) \\
& =\frac{S}{2} \int_{0}^{L} \mathrm{~d} z\left(\rho_{0} U^{2}+\frac{P^{2}}{\gamma \rho_{0} g H}+\frac{\Phi^{2}}{\gamma(\gamma-1) \rho_{0} g H}\right)
\end{aligned}
$$

where the height of cylinder $L$ may tend to plus infinity. It readily follows from Eqs. (1)-(7), that

$$
\begin{aligned}
\frac{\mathrm{d} \varepsilon}{\mathrm{d} t} & =-S \int_{0}^{L} \frac{\partial}{\partial z}\left(p^{\prime} V\right) \mathrm{d} z \\
& =-S \int_{0}^{L} \frac{\partial}{\partial z}(P U) \mathrm{d} z=-\left.S(P U)\right|_{0} ^{L}=0 .
\end{aligned}
$$

For $\varepsilon$ to be constant, there is a certain freedom of establishing of boundary conditions at $z=0$ and $z=L$, for 
example, at any instant $U(z=0)=U(z=L)=0$, or, $U(z=0)=0, P(z=L)=0$. The boundary conditions of the second and third kinds (homogeneous) are also admissible. The boundary conditions should reflect the physical conditions of a flow. The total energy of a flow keeps constant in a close volume of a resonator if there are no internal losses or/and external inflow of energy.

\subsection{Zero velocity at the boundaries}

The wave equation which governs the perturbation of velocity (it corresponds exclusively to the acoustic branches, since the part of the vertical velocity $U$ which specifies the entropy mode, is zero), takes the form in accordance to the dispersion relations $\omega_{1,2}(k)$ (Eqs. (10)):

$$
\frac{\partial^{2} U}{\partial t^{2}}-\gamma g H\left(\frac{\partial^{2} U}{\partial z^{2}}-\frac{U}{4 H^{2}}\right)=0 .
$$

At the lower $(z=0)$ and upper $(z=L)$ boundaries, we set the conditions

$$
U(z=0)=U(z=L)=0 .
$$

Equation (16) may be solved by subdividing spatial and temporal variables. Letting

$$
U(z, t)=Z(z) \cdot \Theta(t),
$$

one arrives at

$$
\begin{aligned}
\frac{1}{\Theta} \frac{\mathrm{d}^{2} \Theta}{\mathrm{d} t^{2}} & =\gamma g H\left(\frac{1}{Z} \frac{\mathrm{d}^{2} Z}{\mathrm{~d} z^{2}}-\frac{1}{4 H^{2}}\right) \\
& =-\gamma g H\left(\lambda^{2}+\frac{1}{4 H^{2}}\right),
\end{aligned}
$$

where $\lambda$ is some constant. Equation (19) in turn determines the spectrum

$$
\lambda_{n}=n \pi / L, \quad n=1,2, \ldots
$$

and the general solution which satisfies the boundary conditions Eq. (17):

$$
\begin{aligned}
V= & \exp (z / 2 H) \cdot U \\
= & \exp (z / 2 H) \cdot \sum_{n=1}^{\infty} \sin \left(\lambda_{n} z\right)\left(A_{n} \sin \left(c t \sqrt{\lambda_{n}^{2}+\frac{1}{4 H^{2}}}\right)\right. \\
& \left.+B_{n} \cos \left(c t \sqrt{\lambda_{n}^{2}+\frac{1}{4 H^{2}}}\right)\right)
\end{aligned}
$$

where

$$
c=\sqrt{\gamma g H}=\sqrt{\gamma \frac{\bar{p}}{\bar{\rho}}}=\sqrt{\gamma T_{0}\left(C_{p}-C_{v}\right)}
$$

designates the speed of infinitely-small magnitude signal in the uniform fluid, and $A_{n}$ and $B_{n}$ denote dimensional constants, measured in units of velocity. From the second and third equations in the set of Eqs. (8), one may conclude that the acoustic excess quantities $p_{a}^{\prime}$ and $\phi_{a}^{\prime}$ are distributed over the domain $[0, L]$ as follows

$$
\begin{aligned}
p_{a}^{\prime}= & \exp (-z / 2 H) \cdot P_{a}=\exp (-z / 2 H) \cdot\left(P_{1}+P_{2}\right) \\
= & \exp (-z / 2 H) \cdot \rho_{0} c \sum_{n=1}^{\infty} \frac{1}{\sqrt{\lambda_{n}^{2}+\frac{1}{4 H^{2}}}} \\
& \cdot\left(\lambda_{n} \cos \left(\lambda_{n} z\right)-\alpha \sin \left(\lambda_{n} z\right)\right) \\
& \cdot\left(A_{n} \cos \left(c t \sqrt{\lambda_{n}^{2}+\frac{1}{4 H^{2}}}\right)\right. \\
& \left.-B_{n} \sin \left(c t \sqrt{\lambda_{n}^{2}+\frac{1}{4 H^{2}}}\right)\right), \\
\phi_{a}^{\prime}= & \exp (-z / 2 H) \cdot \Phi_{a}=\exp (-z / 2 H) \cdot\left(\Phi_{1}+\Phi_{2}\right) \\
= & \frac{(\gamma-1) \rho_{0} g}{c} \exp (-z / 2 H) \cdot \sum_{n=1}^{\infty} \frac{\sin \left(\lambda_{n} z\right)}{\sqrt{\lambda_{n}^{2}+\frac{1}{4 H^{2}}}} \\
& \cdot\left(A_{n} \cos \left(c t \sqrt{\lambda_{n}^{2}+\frac{1}{4 H^{2}}}\right)\right. \\
& \left.-B_{n} \sin \left(c t \sqrt{\lambda_{n}^{2}+\frac{1}{4 H^{2}}}\right)\right)
\end{aligned}
$$

where

$$
\alpha=\left(\frac{1}{\gamma}-\frac{1}{2}\right) \frac{1}{H}
$$

is the positive constant. The total excess pressure is in fact a sum of acoustic and entropy contributions, $p^{\prime}=p_{a}^{\prime}+p_{0}^{\prime}$. The entropy part may be represented by any smooth function of the vertical coordinate, $z$. It refers to zero velocity (and therefore, does not disturb zero boundary conditions), but to the non-zero density perturbations in accordance to Eq. (13). The nodes of acoustic pressure are determined by the equations

$$
\lambda_{n}=\alpha \tan \left(\lambda_{n} z\right), \quad n=1,2, \ldots,
$$

where each of them yields $n$ particular solutions

$\frac{z_{k}}{L}=\frac{\arctan (n \pi /(\alpha L))}{n \pi}+\frac{k}{n}, \quad k=0, \ldots, n-1$.

The nodes of acoustic pressure do not longer coincide with the antinodes of velocity. These last ones are situated in points that are determined by the equations

$$
\lambda_{n}=-\frac{1}{2 H} \tan \left(\lambda_{n} z\right), \quad n=1,2, \ldots,
$$

where

$\frac{z_{k}}{L}=-\frac{\arctan (2 n \pi H / L)}{n \pi}+\frac{k}{n}, \quad k=1, \ldots, n$.

The nodes of pressure are displaced towards the smaller coordinates as compared to the case $g=0$. The 
antinodes of velocity get shifted to the larger coordinates. The distance between nodes in acoustic pressure keeps constant. The nodes of $\phi_{a}^{\prime}$ are located at the same points as those in the profile of velocity. It is remarkable that acoustic pressure no longer possesses antinodes at the ends of the resonator, $z=0$ and $z=L$. The vertical coordinates of nodes of velocity are determined by equalities

$$
\frac{z_{k}}{L}=\frac{k}{n}, \quad k=0, \ldots, n,
$$

and the antinodes of acoustic pressure are established by the following set

$$
\frac{\lambda_{n}}{\gamma H\left(\lambda_{n}^{2}-\alpha / 2 H\right)}=-\tan \left(\lambda_{n} z\right), \quad n=1,2, \ldots
$$

This corresponds to the antinodal points

$$
\begin{aligned}
\frac{z_{k}}{L} & =-\frac{\arctan \left(\frac{\lambda_{n}}{\gamma H\left(\lambda_{n}^{2}-\alpha / 2 H\right)}\right)}{n \pi}+\frac{k}{n}, \\
k & =1, \ldots, n,
\end{aligned}
$$

if $\lambda_{n}^{2}-\alpha / 2 H>0$. These antinodes of pressure are shifted towards the smaller coordinates as compared to the case without external force for any $n$ if $L=H$, but for a large enough $L$, if $\lambda_{n}^{2}-\alpha / 2 H<0$, they get shifted towards larger coordinates. This condition may be satisfied for $L$ about 10 times larger than $H$ in the model of the standard atmosphere.

The limit $g \rightarrow 0$ and therefore $H \rightarrow \infty$ may be readily traced. Note that the product $g H$ remains constant and equals $T_{0}\left(C_{p}-C_{v}\right)$ (in accordance with Eq. (5)), as well as the sound speed $c$ (Eq. (22)). There are $n$ well-known nodes of acoustic pressure in this limit which correspond to the initially uniform medium:

$$
\frac{z_{k}}{L}=\frac{k+1 / 2}{n}, \quad k=0, \ldots, n-1 .
$$

Equations (21), (23) also take the well-known forms:

$$
\begin{aligned}
V= & U=\sum_{n=1}^{\infty} \sin \left(\lambda_{n} z\right)\left(A_{n} \sin \left(c t \lambda_{n}\right)\right. \\
& \left.+B_{n} \cos \left(c t \lambda_{n}\right)\right), \\
\phi_{a}^{\prime}= & \Phi_{a}=0, \\
p_{a}^{\prime}= & P_{a}=\rho_{0} c \sum_{n=1}^{\infty} \cos \left(\lambda_{n} z\right)\left(A_{n} \cos \left(c t \lambda_{n}\right)\right. \\
& \left.-B_{n} \sin \left(c t \lambda_{n}\right)\right) .
\end{aligned}
$$

The nodes of acoustic pressure are located at the points determined by equations $\cos \left(\lambda_{n} z\right)=0$, that is, by Eq. (32), and they coincide with the antinodes of velocity. Figure 1 shows distribution of velocity and acoustic pressure in a resonator for the following data: $g=9.806 \mathrm{~m} / \mathrm{s}^{2}, \gamma=1.4, p_{0}=101325 \mathrm{~Pa}$, a)

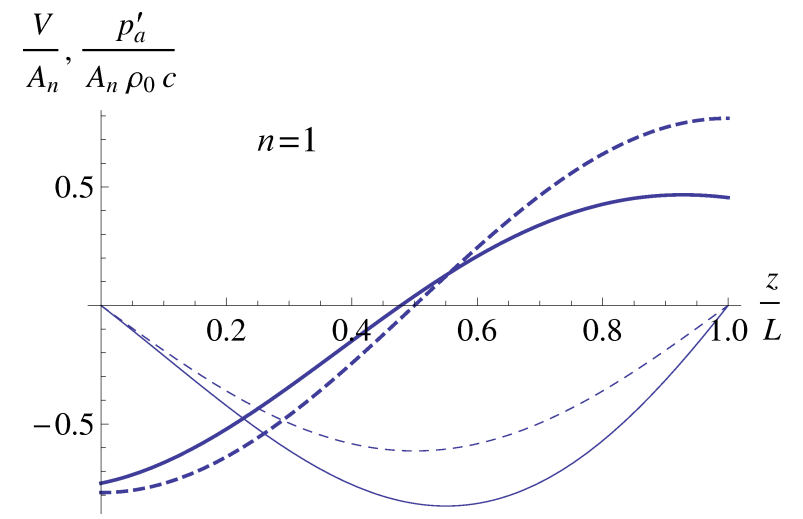

b)

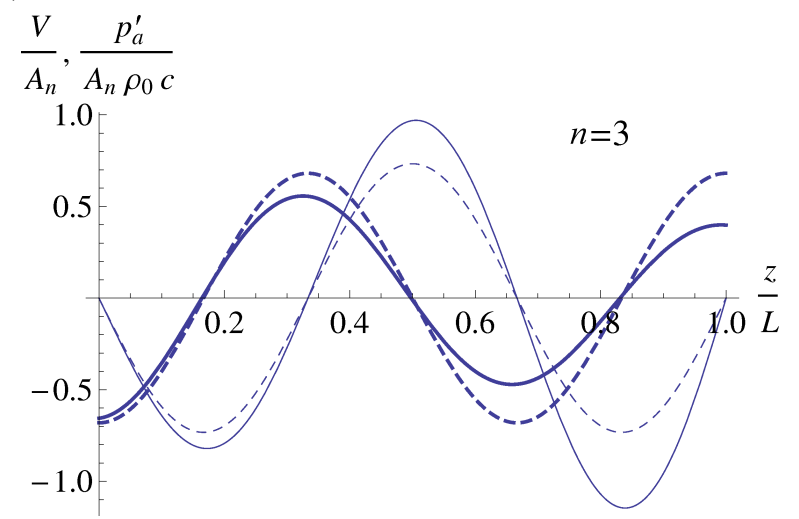

Fig. 1. Velocity (thin lines) and acoustic pressure (bold lines) in a resonator of length $L$ which equals $H, B_{n}=0$ $\left(A_{n} \neq 0\right)$ : a) $n=1, t=30$, b) $n=3, t=60 \mathrm{~s}$. The dotted lines relate to the case without external force $(g=0$, in accordance to Eqs. (33)). Velocity equals zero at the boundaries of a resonator.

$\rho_{0}=1.225 \mathrm{~kg} / \mathrm{m}^{3}$. That corresponds to $H=8435 \mathrm{~m}$. We equate $B_{n}$ zero.

Some stationary perturbations of pressure and $\phi$ which specify stationary entropy mode, are plotted in Fig. 2. They are evaluated in accordance to Eq. (13) and may contribute to the total field in the volume of a resonator on a par with acoustic disturbances. In Fig. 2,

$$
\begin{aligned}
& P_{0}=\exp (0.5) \frac{z}{L}(\mathrm{a}), \\
& P_{0}=\exp (z / 2 L) \frac{z}{L}(\mathrm{~b}), \\
& P_{0}=\cos (\pi \cdot z / L)(\mathrm{c}), \\
& P_{0}=\exp (-z / 2 L) \frac{z}{L}(\mathrm{~d}),
\end{aligned}
$$

$\Phi_{0}$ is determined by Eq. (13), and $p_{0}^{\prime}, \phi_{0}^{\prime}$ relate to $P_{0}$ and $\Phi_{0}$ by means of Eqs. (7). $P_{0}$ may include a factor which is measured in units of pressure. Figures 2 and 4 show the dimensional perturbations divided by their maximum values over the domain $[0, L]$. 
a)

$\frac{\phi_{0}^{\prime}}{K}, \frac{p_{0}^{\prime}}{K}$

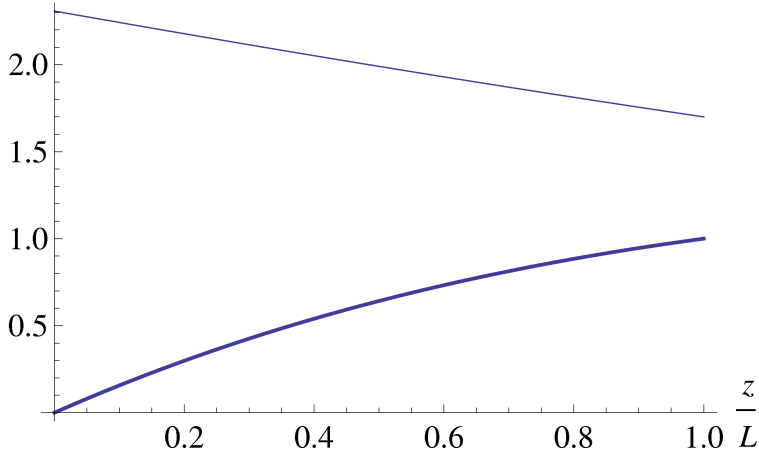

c)

$\frac{\phi_{0}{ }^{\prime}}{K}, \frac{p_{0}{ }^{\prime}}{K}$

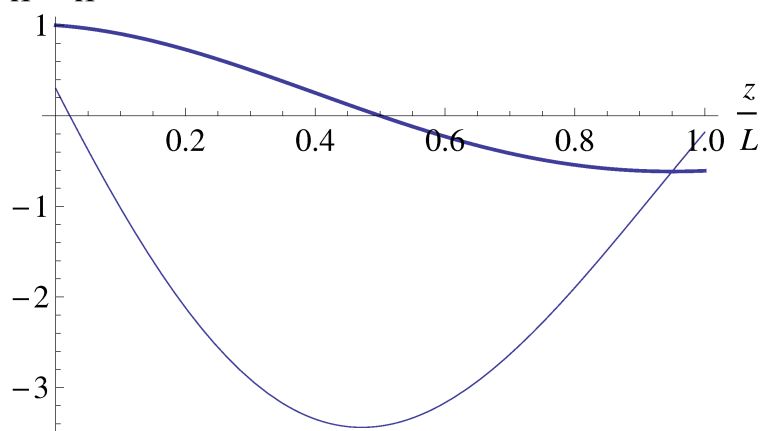

b)

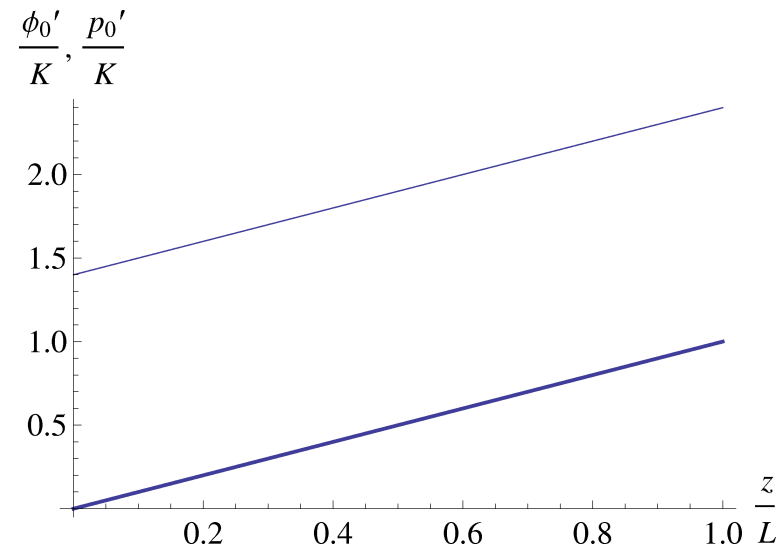

d)

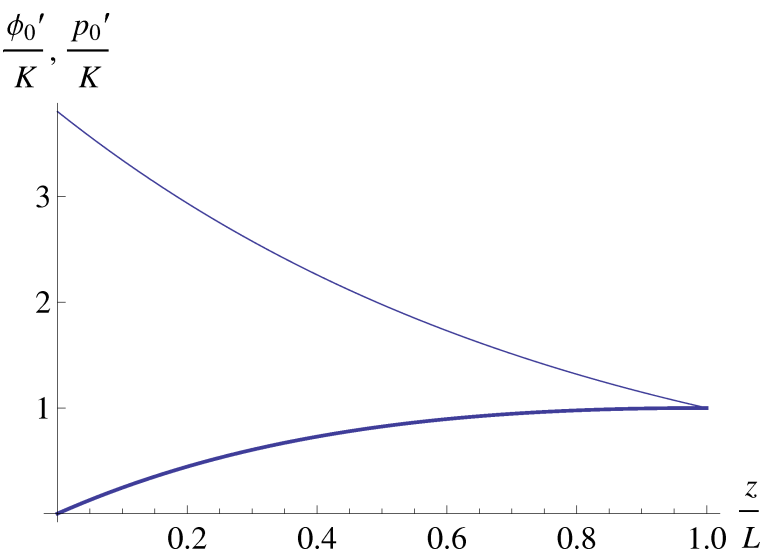

Fig. 2. Exemplary profiles of stationary perturbations in pressure and $\phi$. The bold lines denote perturbation in pressure. $K$ is the absolute value of maximum perturbation of pressure, $p_{0}^{\prime}$, over the domain $z \in[0, L] . P_{0}$ at all figures is determined by Eq. (34), and $\Phi_{0}$ is evaluated in accordance to Eq. (13). with

It may be concluded that the entropy disturbances

$$
\begin{aligned}
p_{0}^{\prime}= & \left(\frac{p_{0} \Delta T}{H T_{0}} z+C\right) \exp (-z / H), \\
\phi_{0}^{\prime}= & \frac{p_{0} \Delta T}{H T_{0}}(z(1-\gamma)+H) \exp (-z / H) \\
& +C(1-\gamma) \exp (-z / H),
\end{aligned}
$$

where $C$ is any constant, yield the constant excess temperature, $\Delta T$, over the whole interval. $C=0$ is the case of Fig. 2d.

\subsection{Zero pressure disturbances at the boundaries}

The equation which governs perturbation of acoustic pressure takes the form similar to Eq. (16):

$$
\frac{\partial^{2} P_{a}}{\partial t^{2}}-\gamma g H\left(\frac{\partial^{2} P_{a}}{\partial z^{2}}-\frac{P_{a}}{4 H^{2}}\right)=0
$$

At the upper and lower boundaries, we set the conditions

$$
P_{a}(z=0)=P_{a}(z=L)=0 .
$$

Repeating the evaluations described in the previous subsection, we finally arrive at solution which satisfies the boundary conditions (37):

$$
\begin{aligned}
p_{a}^{\prime}= & \exp (-z / 2 H) \cdot P_{a} \\
= & \exp (-z / 2 H) \cdot \sum_{n=1}^{\infty} \sin \left(\lambda_{n} z\right) \\
& \cdot\left(C_{n} \sin \left(c \sqrt{\lambda_{n}^{2}+\frac{1}{4 H^{2}}} t\right)\right. \\
& \left.+D_{n} \cos \left(c \sqrt{\lambda_{n}^{2}+\frac{1}{4 H^{2}}} t\right)\right),
\end{aligned}
$$

where $C_{n}$ and $D_{n}$ are constants measured in units of pressure. The velocity field may be established from the second equation from Eqs. (8), and $\Phi_{a}$ from the third equation from this set: 


$$
\begin{aligned}
V= & \exp (z / 2 H) \cdot U_{z}=\frac{\exp (z / 2 H)}{\rho_{0} c} \sum_{n=1}^{\infty} \frac{\sqrt{\lambda_{n}^{2}+\frac{1}{4 H^{2}}}}{\lambda_{n}^{2}+\alpha^{2}} \\
& \cdot\left(\lambda_{n} \cos \left(\lambda_{n} z\right)+\alpha \sin \left(\lambda_{n} z\right)\right) \\
& \cdot\left(C_{n} \cos \left(c \sqrt{\lambda_{n}^{2}+\frac{1}{4 H^{2}}} t\right)\right. \\
& \left.-D_{n} \sin \left(c \sqrt{\lambda_{n}^{2}+\frac{1}{4 H^{2}}} t\right)\right), \\
\phi_{a}^{\prime}= & \exp (-z / 2 H) \cdot \Phi_{a}=-\frac{(\gamma-1) g \exp (-z / 2 H)}{c^{2}} \\
& \cdot \sum_{n=1}^{\infty} \frac{1}{\lambda_{n}^{2}+\alpha^{2}}\left(\lambda_{n} \cos \left(\lambda_{n} z\right)+\alpha \sin \left(\lambda_{n} z\right)\right) \\
& \left(C_{n} \sin \left(c \sqrt{\lambda_{n}^{2}+\frac{1}{4 H^{2}}} t\right)\right. \\
& \left.+D_{n} \cos \left(c \sqrt{\lambda_{n}^{2}+\frac{1}{4 H^{2}}} t\right)\right) .
\end{aligned}
$$

The nodes of velocity are determined by the equations $\lambda_{n}=-\alpha \tan \left(\lambda_{n} z\right), \quad n=1,2, \ldots$,

$\frac{z_{k}}{L}=-\frac{\arctan (n \pi /(\alpha L))}{n \pi}+\frac{k}{n}, \quad k=1, \ldots, n$,

and the antinodes of acoustic pressure by the equations

$\lambda_{n}=\frac{1}{2 H} \tan \left(\lambda_{n} z\right), \quad n=1,2, \ldots$,

$\frac{z_{k}}{L}=\frac{\arctan (2 n \pi H / L)}{n \pi}+\frac{k}{n}, \quad k=0, \ldots, n-1$.

In turn, the following set represents antinodes of velocity:

$$
\begin{gathered}
\frac{\lambda_{n}}{\gamma H\left(\lambda_{n}^{2}-\alpha / 2 H\right)}=\tan \left(\lambda_{n} z\right), \quad n=1,2, \ldots, \\
\frac{z_{k}}{L}=\frac{\arctan \left(\frac{\lambda_{n}}{\gamma H\left(\lambda_{n}^{2}-\alpha / 2 H\right)}\right)}{n \pi}+\frac{k}{n}, \\
k=0, \ldots, n-1,
\end{gathered}
$$

if $\lambda_{n}^{2}-\alpha / 2 H>0$. In the limit $g \rightarrow 0$, Eqs. (38), (39) may be rearranged into expressions

$$
\begin{aligned}
p_{a}^{\prime}= & P_{a}=\sum_{n=1}^{\infty} \sin \left(\lambda_{n} z\right)\left(C_{n} \sin \left(c t \lambda_{n}\right)\right. \\
& \left.+D_{n} \cos \left(c t \lambda_{n}\right)\right), \\
\phi_{a}^{\prime}= & \Phi_{a}=0 \\
V= & U=\frac{1}{\rho_{0} c} \sum_{n=1}^{\infty} \cos \left(\lambda_{n} z\right)\left(C_{n} \cos \left(c t \lambda_{n}\right)\right. \\
& \left.-D_{n} \sin \left(c t \lambda_{n}\right)\right),
\end{aligned}
$$

with the nodes of acoustic pressure correspondent to antinodes of velocity and vice versa. Figure 3 shows distribution of acoustic pressure and velocity in the sound wave at $n=1, t=30 \mathrm{~s}$ (the case $a$ ), and $n=3$, $t=60 \mathrm{~s}$ (the case $b$ ). It represents curves which corresponding to the cases with external force and without it.

a)

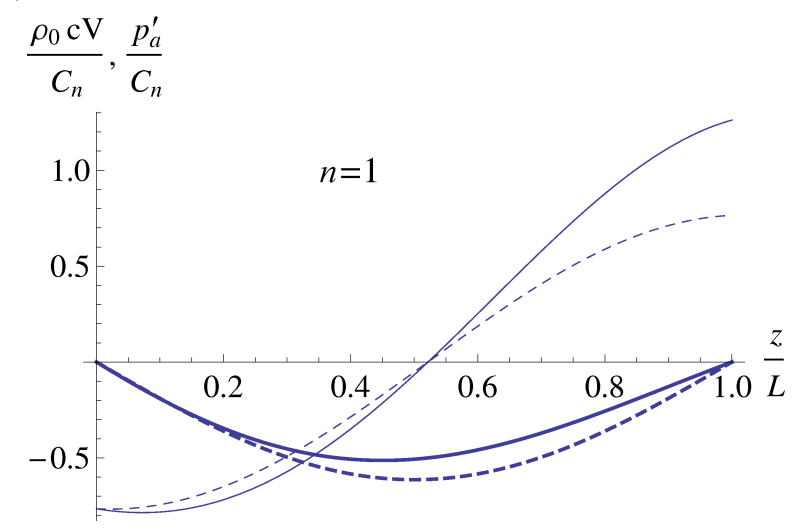

b)

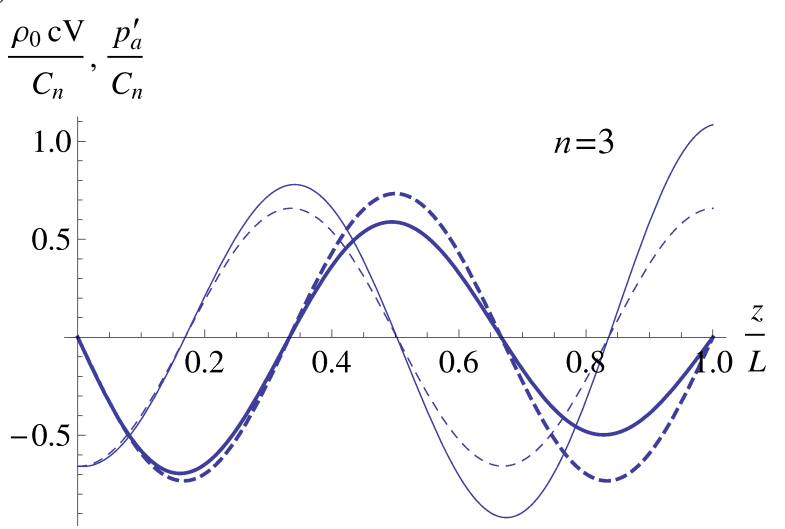

Fig. 3. Velocity (thin lines) and acoustic pressure (bold lines) in a resonator of length $L$ which equals $H, D_{n}=0$, $C_{n} \neq 0$ : a) $n=1, t=30 \mathrm{~s}$, b) $n=3, t=60 \mathrm{~s}$. The bold lines denote perturbations in pressure. Acoustic pressure equals zero at the boundaries of a resonator. The dotted lines relate to the case without external force $(g=0$, in accordance to Eqs. (43)).

The exemplary perturbations in the entropy mode with zero pressure at the boundaries $z=0$ and $z=L$ are plotted in Fig. 4, where

$$
\begin{aligned}
& P_{0}=\sin (\pi \cdot z / L) \quad(\mathrm{a}), \\
& P_{0}=\sin (2 \pi \cdot z / L) \quad(\mathrm{b}),
\end{aligned}
$$

$\Phi_{0}$ is determined by Eq. (13), and $p_{0}^{\prime}, \phi_{0}^{\prime}$ relate to $P_{0}$ and $\Phi_{0}$ by the relations Eq. (7). $P_{0}$ may include a factor which is measured in units of pressure. They may be added to acoustic perturbation with zero pressure at the boundaries forming a new field in the volume of a resonator. 
a)

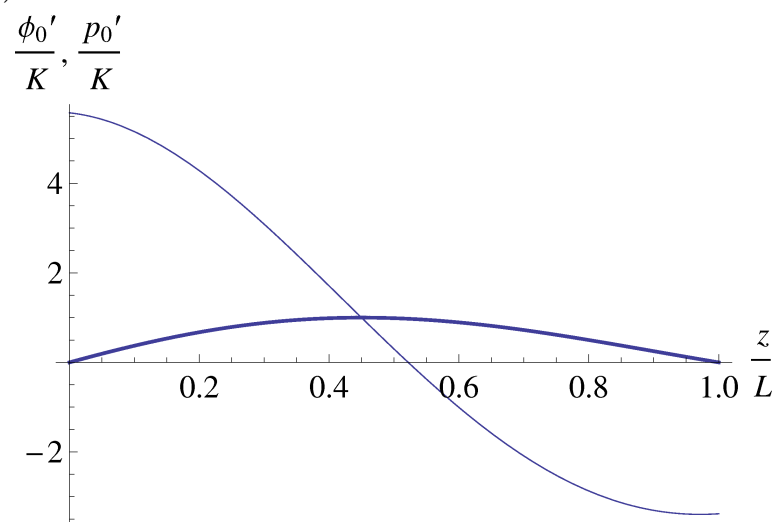

b)

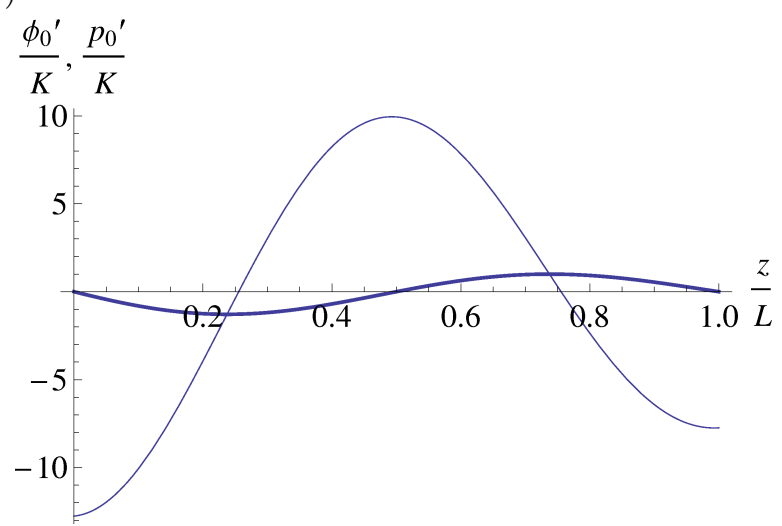

Fig. 4. Exemplary profiles of stationary perturbations of pressure and $\phi$ in the stationary mode. The bold lines denote perturbation in pressure. $\mathrm{K}$ is the absolute value of maximum perturbation of pressure over the domain $z \in[0, L] . P_{0}$ is determined by Eq. (44), and $\Phi_{0}$ is calculated in accordance to Eq. (13).

\section{Concluding remarks}

Any perturbations in a resonator which satisfy physically justified boundary conditions follow from the relations linking variables $U, P$, and $\Phi$ in sound and entropy modes which may exist in a closed volume of a gas affected by the constant mass force. Actually, every mode which represents a root of the dispersion equation, possesses individual relations of specific thermodynamic perturbations. The exact links of excess pressure, mass density, and velocity in travelling waves in unbounded volumes of a gas are integrodifferential, they have been derived exactly with regard to one-dimensional flow in (Leble, Perelomova, 2013; Perelomova, 1998). The approximate relations in the case of short perturbations (as compared to $H$ ) have been derived by the author in (PERELOMOVA, 2009). We consider a resonator bounded by planes $z=0$ and $z=L$. Without restrictions, the lower boundary may be situated at any other plane.

As far as the author knows, the standing waves in a fluid which is affected by a mass force, are a new sub- ject of investigations. The case of constant mass force is the most simple and makes it possible to use elementary methods of mathematical physics in a theoretical analysis of perturbations in a volume of a resonator. This study is devoted to an ideal inviscid gas. It may be readily generalised in the case of a fluid different from an ideal gas, including liquid, replacing $\gamma$ by $c^{2} \rho_{0} / p_{0}$. In particular, $\gamma>2$ corresponds to negative $\alpha$ in accordance to Eq. (24). The nonlinearity and attenuation in conservation equations were not taken into account. Nonlinear resonators containing homogeneous fluid with Newtonian attenuation have been considered by Kaner and co-authors in (KANER et al., 1977) by means of the multi-scale method. It has been established that under some conditions (periodic perturbations, zero on average acoustic pressure, weak nonlinearity), the acoustic waves which travel in opposite directions do not interact in a volume of resonator and may be considered independently. The important property of standing acoustic waves in a fluid with attenuation is that the nodes of acoustic pressure and velocity are no longer constantly situated but alter their coordinate with time. This is conditioned by the dispersion relation of linear flow with attenuation which does not permit a solution in the separated temporal and spacial coordinates. The correspondent one-dimensional equation (the linear analogue of the Burgers equation) takes the form (HAMilton, Blackstock, 1997):

$$
\left(\frac{\partial^{2} p_{a}^{\prime}}{\partial t^{2}}-c^{2} \frac{\partial^{2} p_{a}^{\prime}}{\partial x^{2}}\right)-\frac{\delta}{c^{2}} \frac{\partial^{3} p_{a}^{\prime}}{\partial t^{3}}=0
$$

where $\delta$ denotes the diffusity of sound. As for the considered in this study fluid, affected by an external force, the form of dispersion relations for acoustic branches, Eq. (10), and relative linear equations Eqs. (16), (36) allow to search a solution in the separated variables. In view of that, nodes and antinodes of perturbations are situated permanently, although differently than in the case of the homogeneous background pressure and mass density. Non-linear waves in the stratified media which propagate in the waveguides and some special methods to describe the relative field may be found in the textbook (LEBLE, 1990).

Two kinds of boundary conditions are considered: with zero velocity at the upper and lower boundaries (Subsec. 3.1) and with zero perturbations in pressure there (Subsec. 3.2). As for the first case, no stationary smooth pressure field which corresponds to the entropy mode, disturbs the boundary conditions and may contribute to the standing acoustic wave. As for the second case, the entropy mode should yield zero pressure perturbation at the boundaries of a resonator. The entropy mode may change the sound speed by means of variations in temperature inherent to this mode. Stationary perturbations which correspond to increase in the temperature, $\Delta T$, make the local sound speed in gases to enlarge. The nonlinear losses in a thermo- 
viscous medium lead to acoustic heating, that is, to the entropy mode which slowly varies in time. Hence, the spacial spectrum of travelling waves slowly varies in time as well. The nonlinear heating is noticeable for large acoustic perturbations, for processes which occur for a long time, and in the closed volumes of highly viscous fluids.

The positions of nodes and antinodes of acoustic pressure and velocity in both types of boundary conditions are evaluated. The nodes of pressure do not coincide with antinodes of velocity, and vice versa. They differ from nodes and antinodes in a resonator containing a gas which is not affected by an external force. This conclusion also concerns the ends of a resonator. The distance between nodes and antinodes of any perturbation in a standing wave keeps constant. The theory may have a practical application. It may be useful to conclude about an external force remotely basing on the observation of perturbations or to govern nodes and antinodes in medical applications accurately.

As for the frequencies of the standing waves, they are determined by Eqs. (10) and wavenumbers which are inherent to the resonator. The smallest wave number $k$ corresponds $n=1$ and equals $\pi / L$. There is the cut-off frequency, that is, minimal frequency of standing waves

$$
\omega_{\min }=c \sqrt{\frac{\pi^{2}}{L^{2}}+\frac{1}{4 H^{2}}} .
$$

In the case of $c=340 \mathrm{~m} / \mathrm{s}$ and $H=L=8435 \mathrm{~m}$, $\omega_{\min }=0.13 \mathrm{~Hz}$, that belongs to the infrasound region. One may readily evaluate minimal $n$ which corresponds to the audio frequency, that is, exceeds $20 \mathrm{~Hz}$. It equals 158. In general, the theory gives noticeably different results (referring to the case of the uniform fluid) if the characteristic wave numbers of perturbations are comparable to or are lower than the inverse scale of stratification, $H^{-1}$. That in turn depends on $g$ and $T_{0}$.

\section{References}

1. Brekhovskikh L.M., Godin A.O. (1990), Acoustics of layered media, Springer-Verlag, Berlin.

2. Chu B.-T., Kovasznay L.S.G. (1958), Nonlinear interactions in a viscous heat-conducting compressible gas, J. Fluid. Mech., 3, 494-514.

3. Eckart C. (1960), Hydrodynamics of Oceans and Atmospheres, Pergamon Press, London.

4. Hamilton M.F., Blackstock D.T. [Eds.] (1997), Nonlinear Acoustics, Academic Press, San Diego.

5. Jones R.M. (2001), The dispersion relation for internal acoustic-gravity waves in a baroclinic fluid, Physics of Fluids, 13, 1274-1280.

6. Kaner A., Rudenko O.V., Khokholov R.V. (1977), Theory of nonlinear oscillations in acoustic resonators, Sov. Phys. Acoust., 23, 5, 432-437.

7. Leble S.B. (1990), Nonlinear waves in waveguides with stratification, Springer-Verlag, Berlin.

8. Leble S., Perelomova A. (2013), Problem of proper decomposition and initialization of acoustic and entropy modes in a gas affected by the mass force, Applied Mathematical Modelling, 37, 629-635.

9. Pedloski J. (2006), Geophysical fluid dynamics, Springer-Verlag, Berlin.

10. Perelomova A. (1998), Nonlinear dynamics of vertically propagating acoustic waves in a stratified atmosphere, Acta Acustica, 84, 1002-1006.

11. Perelomova A. (2006), Development of linear projecting in studies of non-linear flow. Acoustic heating induced by non-periodic sound, Physics Letters A, 357, $42-47$.

12. Perelomova A. (2009), Weakly nonlinear dynamics of short acoustic waves in exponentially stratified gas, Archives of Acoustics, 34, 2, 197-213.

13. Rudenko O.V., Soluyan S.I. (1977), Theoretical foundations of nonlinear acoustics, Plenum, New York. 